\title{
Acceptability of community-based adherence clubs among health facility staff in South Africa: a qualitative study
}

This article was published in the following Dove Press journal:

Patient Preference and Adherence

II September 2017

Number of times this article has been viewed

\author{
Ndumiso Tshuma ${ }^{1,2}$ \\ Ofentse Mosikare ${ }^{1,2}$ \\ Jessica A Yun' \\ Olufunke A Alaba ${ }^{3}$ \\ Meera S Maheedhariah ${ }^{4}$ \\ Keith Muloongo, ${ }^{1,2}$ \\ Peter S Nyasulu $2,5,6$ \\ 'Community AIDS Response, \\ Johannesburg, South Africa; ${ }^{2}$ School of \\ Health Sciences, Monash University \\ South Africa, Johannesburg, South \\ Africa; ${ }^{3} \mathrm{School}$ of Public Health and \\ Family Medicine, University of Cape \\ Town, South Africa; ${ }^{4}$ Department \\ of Human Behaviour, College of \\ South Nevada and University of \\ California, Los Angeles, CA, USA; \\ ${ }^{5} \mathrm{~S}$ chool of Public Health, Faculty \\ of Health Sciences, University of \\ the Witwatersrand, Johannesburg, \\ South Africa; ${ }^{6}$ Department of Global \\ Health, Division of Epidemiology \\ and Biostatistics, Faculty of Medicine \\ and Health Sciences, Stellenbosch \\ University, Cape Town, South Africa
}

Correspondence: Peter S Nyasulu Department of Global Health, Division of Epidemiology and Biostatistics, Faculty of Medicine and Health Sciences, Stellenbosch University, Cape Town, South Africa

Tel +27 2I 9389157

Email pnyasulu2sun.ac.za
Background: Patient retention in care for HIV/AIDS is a critical challenge for antiretroviral treatment programs. Community-based adherence programs (CBAPs) as compared to health care facility-based adherence programs have been considered as one of the options to provide treatment maintenance support for groups of patients on antiretroviral therapy. Such an approach provides a way of enhancing self-management of the patient's condition. In addition, CBAPs have been implemented to support antiretroviral treatment expansion in resource-limited settings. CBAPs involve 30 patients that are allocated to a group and meet at either a facility or a community venue for less than an hour every 2 or 3 months depending on the supply of medication. Our study aimed to establish perceived challenges in moving adherence clubs from health facilities to communities.

Methods: A qualitative study was conducted in 39 clinics in Mpumalanga and Gauteng Provinces in South Africa between December 2015 and January 2016. Purposive sampling method was used to identify nurses, club managers, data capturers, pharmacists and pharmacy assistants who had been involved in facility-based treatment adherence clubs. Key-informant interviews were conducted. Also, semi-structured interviews were used and thematic content analysis was done.

Results: A total of 53 health care workers, 12 (22.6\%) males and 41 (77.4\%) females, participated in the study. Most of them 49 (92.5\%) indicated that participating in community adherence clubs were a good idea. Reduction in waiting time at the health facilities, in defaulter rate, improvement in adherence to treatment as well as reduction in stigma were some of the perceived benefits. However, security of medication, storage conditions and transportation of the prepacked medication to the distribution sites were the areas of concern.

Conclusion: Health care workers were agreeable to idea of the moving adherence clubs from health facilities to communities. Although some challenges were identified, these could be addressed by the key stakeholders. However, government and nongovernmental organizations need to exercise caution when transitioning to community-based adherence clubs.

Keywords: adherence clubs, antiretroviral therapy, HIV, health facility, chronic clubs, cohorts, patient retention

\section{Background}

It is estimated that in 2015, 6.19 million people were living with HIV in South Africa. ${ }^{1}$ The South Africa National Department of Health National Adherence Guidelines Strategy was launched in $2016 .^{2}$ Adherence clubs were established to cater for individuals who are clinically stable on antiretroviral treatment (ART) or other chronic medication for noncommunicable diseases. ${ }^{2,3}$ Adherence club programs are a community health 
care worker (CHW)-facilitated model of care to increases awareness and understanding about self-management of individual patients' conditions. ${ }^{3}$ For purposes of this study, the term "adherence clubs", also known as ART treatment clubs, clubs, community-based adherence groups, communitybased adherence support and cohorts, will be used. ${ }^{3-7}$

Adherence clubs is a recommended strategy that supports treatment compliance, retention in care and expansion of HIV care services in resource-limited settings. ${ }^{3,8,9}$ Despite the high ART retention in formal health care facilities, many HIV-positive people struggle with frequent appointments to health care facilities and may drop out of care entirely. ${ }^{9}$ In addition, high treatment-related costs for ART, shortage of professional health care workers and lack of access to high quality care compromise the ability of patients to receive HIV treatment in resource-limited regions. ${ }^{10-13}$ However, data are limited regarding the effectiveness of different intervention strategies that promote retention in care especially in the South African context. ART has modified the natural history of HIV infection turning it into a chronic infection requiring long-term clinical management. ${ }^{14-16}$ As such adherence clubs are designed to support the management of HIV infection and other chronic diseases. ${ }^{4}$

Adherence clubs are a club comprising between 25 and 30 patients who meet every 2 months led by a CHW, lay counselor or peer educators. ${ }^{3,8,17}$ The program entails a group counseling session (such as safe conception, importance of retention, etc.), distribution of prepacked ART medication at a central pharmacy and a brief symptom screening which is all to be completed in 60 minutes. ${ }^{3,6,7,9}$ A clinic consultation led by a nurse occurs twice every year and is repeated annually. ${ }^{9}$ With regard to ART medication, patients are required to pick up their prepacked ART medication every 2 months at the community-based adherence club visits. ${ }^{3}$

The main concept of community-based adherence clubs is to support clinically "stable" patients who have been on ART for $>12$ months. This was a way to decongest facilities by shifting ART medication collections and consultations to clubs. ${ }^{3,6,7,9}$ Furthermore, patients who have had consecutive two undetectable viral loads with viral load measurement done after 6 months of treatment initiation and did not have frequent follow-up visits due to medical conditions were considered eligible to attend the program. ${ }^{3}$ Patients attending clinical consultations who met the criteria for adherence clubs support were referred to join the adherence clubs program.

The adherence clubs strategy and the group-based model of care have shown potential benefits associated with reduced loss to follow-up of patients on ART, decline in mortality as well as improved viral suppression..$^{2,7,17}$ Recent studies have reported that the main benefits of rapid ART provision and community-based adherence clubs are the reduction in time taken to access medication and cost saving on transportation associated with frequent clinic visits. ${ }^{9,18,19}$ Furthermore, there was no difference in mortality among those who were attending adherence clubs $4.9 \%$ versus $6.3 \%$, respectively. ${ }^{5}$ This was due to patients having an improved knowledge about HIV/AIDS, thus providing an opportunity for patients to overcome stigma and denial after a clear understanding of the relevance of treatment adherence. ${ }^{5}$

Despite success reported, one of the key obstacles in the implementation of adherence clubs programs is the need to have a reliable and steady supply of medications. For example, the Ministry of Health in Malawi experienced temporary drug "stock out"., 3 This scenario led to a decline in a number of patients enrolling in the adherence club programs. As such, this requires the close cooperation of the Ministry of Health due to the issue that they will have to consider the communitybased adherence program in their wage bill envelopes with other existing programs that face remuneration difficulties. ${ }^{9}$ This includes the costs of maintaining community venues for the adherence clubs as well as transportation costs of materials for the clubs. ${ }^{3,19}$ To expand an effective health care program, patients and health care professionals need to change their mindset and adapt to the management of disease outside of traditional model of clinical care. , $^{3,7}$

Recent studies have focused on the health care facility benefits, the improved clinical outcomes and other patient benefits of implementing adherence clubs and group-based models of care. ${ }^{7,20,21}$ Potential confounders that would mask the effect of adherence clubs have been highlighted as patient's preferences to enroll in the clubs are based on the availability of the clubs. ${ }^{7,22}$ However, implementation of a transition to communities is contingent on engaging with health facility staff so as to overcome challenges that would impede implementation of the community-based adherence clubs. ${ }^{23,24}$ A previous study that used routine data from Malawi, South Africa, DRC and Mozambique on community-supported models showed that ART delivery is context specific. It is also dependent on a number of factors such as the extent of service decentralization, task shifting, HIV prevalence, the availability of safe and simple ART regimens, health care services capacity and regulatory or logistical constraints to ART delivery. ${ }^{9}$

Another study conducted in Mozambique identified the limited access to community ART groups as a threat to the national rollout of the community-based adherence 
clubs. ${ }^{9}$ There is also a need to establish the acceptability of community-based adherence clubs from a health facility staff perspective. ${ }^{8,9}$ This study aimed to assess acceptability, enablers and barriers to rolling out community-based clubs.

\section{Methods Study design}

This was a qualitative exploratory study using in-depth interviews. The use of in-depth interviews has been shown to be effective in collecting information about how health care workers would like community-based clubs to be implemented in their settings. The study also explored possible challenges in transitioning from health facility-based adherence clubs to community-based adherence clubs among health workers that were involved in the operations of adherence clubs. Participants freely expressed their opinions on the implementation of community-based adherence clubs.

\section{Study setting}

This study was conducted in 39 health facilities in two provinces in South Africa. A total of 13 facilities were in Ehlanzeni District, Mpumalanga Province; 10 health facilities were in the Johannesburg Health District and 16 health facilities in the City of Ekurhuleni, Gauteng Province. The selection of the health facilities was based on the fact that these facilities were already implementing health facilitybased adherence clubs.

\section{Data collection}

Data were collected using a paper-based open-ended questionnaire and were entered into a data collection system designed on personal computers. Two research assistants with experience in conducting qualitative interviews visited 39 health facilities and conducted one-on-one interviews with study participants. Prior to data collection, a program was drawn up and potential participants were notified of the dates and times when the interviews were scheduled to take place. Data collectors were trained in interviewing skills and piloted the questionnaire by conducting six interviews with individual members of the team to assess user-friendliness of the designed data collection tool. Observed limitations with the questionnaire were modified accordingly prior to the main study. This exercise improved the quality of the designed data collection instrument as well as interviewing skills.

\section{Key informant interviews}

The participants were asked questions regarding the idea of transitioning adherence clubs from the health facilities to the communities. In addition, the research assistants probed details about the reasons surrounding the agreement or disagreement with the suggested transition of adherence clubs to communities.

\section{Recruitment of participants}

Health facilities that were implementing health facility-based adherence clubs supported by Community AIDS Response (CARe) were selected. CARe is a regional nongovernmental organization supporting HIV prevention, treatment and care and support programs in communities and health facilities. The selected facilities have been supported by CARe to implement the health facility-based adherence clubs for 22 months. Since the program was implemented in the year 2014, all study participants were involved in the process of conceptualization and implementation of the health facility-based adherence clubs. Study participants were purposively selected so as to ensure representation of the different categories of health facility staff who were directly involved in the health facility-based adherence clubs.

The study participants included a pharmacist, a pharmacy assistant, a data capturer, a sister-in-charge, a club manager and a club sister. Within the clubs, the club manager or club sister was responsible for the day-to-day operations of the adherence clubs. Other responsibilities included supervising the patients during their visits for blood tests, clinical consultations, the signing of files and completion of prescription scripts for patient's medication.

The pharmacists and pharmacist assistants were responsible for ensuring that patients receive their prepacked medication. In some health facilities, nurses prepacked the medication. The sister-in-charge had the role of ensuring that the adherence club program is successfully implemented in the health facility. All study participants were appropriately exposed to adherence clubs and served as key informants' of the study.

\section{Data analysis and management}

The questionnaires were reviewed and coded for unifying ideas by three of the authors to ensure reliability and trustworthiness of the data. In situations where there were observed disagreements in the creation of themes, a vote was taken. Content analysis was used to analyze the data which entailed the reading and texts reviewing of the entire interview. This was to ensure that meaningful units were identified which were condensed into categories and themes based on a priori formulated themes: 1) patient benefits, 2) health system benefits and 3 ) other emerging themes and subthemes that emerged from the interviews. 


\section{Ethical considerations}

Monash University Human Research Ethics Committee approved the study: approval number CF14/28032014001558. All participants were informed about the objectives of the study, their freedom to participate or withdraw from the study at any time if they so wished, confidentiality of information and possible risks and benefits of the study. Verbal informed consent was obtained at the time of data collection which was kept anonymous.

\section{Results}

A total of 53 health care workers were interviewed, and of these participants, $12(22.6 \%)$ were males. Most of the participants 49 (92.5\%) were in favor of bringing communitybased adherence clubs to the communities as they thought this would increase accessibility of the services but also will be more acceptable as community members would view the program as part of their own.

\section{Acceptability of community-based health clubs}

The participants indicated that community-based clubs would be favorable to both the patients and the health care workers as the program will help to reduce the waiting time to collect medication and provide a platform more convenient to pick up medications. This will eventually support patients' compliance to medication, thus reducing the rate of treatment default.

The participants also indicated that the implementation of the community-based adherence clubs might also translate to a reduction in stigma levels, especially among men as they are often uncomfortable attending adherence clubs at a health facility. From a system perspective, the participants indicated that the implementation of the community-based adherence clubs will help decongest the health facilities, release pressure of health care workers as well as free up space for an effective health service delivery to patients coming for services in these clinics.

We have grouped the key findings into "proponents" and "antagonist" of the transition from facility- to communitybased adherence clubs. The proponent group is further divided into "patient benefits" and "health system benefits" with specifically identified themes.

\section{Proponents for establishing community- based adherence clubs}

In support of establishing community-based adherence clubs, participants who supported this shift provided the following reasons in support of this change.

\section{Patient benefits}

The benefits that can be attributed to community-based adherence clubs fall under the following themes: reduction in waiting time, convenience, reduction in defaulter rate, improvement in adherence, and finally reduction in stigma level.

\section{Reduction in waiting time}

The participants were quick to say that the implementation of the community-based adherence clubs would reduce the waiting times in the health facilities as patients will not have to stand in the long queues waiting to pick up their medication. This could eventually improve patient satisfaction in health care services. One sister-in-charge indicated that:

The patients that have been attending the clinic for a long time have expectations. One of those expectations is that they want to be reviewed immediately and go back home or work. Community-based clubs will be able to fulfil this expectation.

A Club Manager in one of the clinics highlighted that if the systems and strategies that were being implemented improved the smooth running of the adherence clubs at the community level. The initiative of transitioning adherence clubs to the communities will lead to a reduction in several people attending public health care facilities. The manager said that:

Should the programme have ideal systems and strategies in place, community-based clubs would certainly be a good idea for our facilities as it will assist in reducing the queues.

\section{Convenience}

Participants reiterated that community-based adherence clubs will be convenient to patients with regard to the reduction in waiting times.

Yes, community-based adherence clubs are a good idea because most patients do not like to come to the clinic as they wait for a long time before they are attended to. The clubs will help to reduce patients waiting time and allow for easy access to medication. (Sister-in-charge)

The participants alluded to the fact that the patients were receiving their medication at a convenient place and that environment alone would enhance their will power to adhere to their medication. The participants showed that there is a relationship between the proximity of the pickup 
point and the levels of adherence to the medication. Among the participants, one was quoted saying:

I believe that if chronic patients are in communities and not within the health facility they may be more compliant with their medication because the community-based clubs will be more convenient for them. Such programmes will also help to reduce our workload as health facility staff. (Club nurse)

Some participants indicated that the convenience of picking medication from the community-based adherence clubs will lead to a reduction in the numbers of club members sending their friends and family members to pick up the medication. A pharmacist and sister-in-charge in two distinct facilities gave the following account:

Patients won't default as often and it will assist patients to access services more conveniently. HIV and AIDS patients will also stop sending their friends as they will come and collect their own medication personally. (Pharmacist)

Yes, community-based adherence clubs would be a good idea as the clubs will encourage clients to honour their appointments without any complaints. (Sister-in-charge)

The convenience of the pickup point was also linked to the fact that it will reduce the financial constraints associated with travel to the health facilities. The patients will have the opportunity to pick up their medication from a walking distance to the community-based adherence club sites, which are in close proximity to the patient's place of residence.

Yes, community-based adherence clubs are a good idea as they will help reduce financial constraints that patients have and they will provide easier access to medication. (Club nurse)

\section{Patient empowerment and self-management}

The participants also associated the formation of the community-based adherence clubs with the ease of formation of community-based patient support structures in the form of support groups. One of the Club Nurses indicated the following:

Yes, community-based adherence clubs are a good idea. It becomes easy to form support groups outside a clinic. Patients will also be able to empower themselves and they will have easy access to treatment. (Club nurse)

\section{Reduce stigma}

The participants highlighted that the implementation of community-based adherence clubs will translate to a reduction in stigma levels and a reduction in fear to pick up medication among patients. Some participants explained the situation as:

It is common that ART patients, men especially, fear attending the health facility for their ART treatment and consultations as they feel there is the stigma associated with being HIVpositive. Perhaps a community-based environment would be much friendlier for clients. (Sister-in-charge)

Yes, community-based clubs would be a good idea. It will help us to avoid the issue of stigma because some patients are still uncomfortable in coming to the clinic to collects their medication. (Data capturer)

\section{Health system benefits}

The second broad category of benefits stated by participants who were in support of the idea to implement communitybased adherence clubs was a health system benefit.

\section{Decongestion of health facilities}

Most of the participants $38(72 \%)$ indicated that there are a growing number of patients receiving health services from the clinic yet the facilities are not expanding in size. This emphasizes the fact that there has been an increase in clinic congestion. The implementation of communitybased clubs was highlighted as a strategy that will translate to alleviating the space constraints and crowding, as clinically stable patients will collect their medication from the community.

Yes, community-based clubs would be a good idea. Our clinic is overcrowded and we have no capacity to extend it. It is not necessary for stable patients to go to the clinic every month. (Sister-in-charge)

Yes, community-based clubs would be a good idea. We have space constraints in the health facility. (Club sister)

One of the sisters in charge linked the decongestion of the health facilities to a reduction in the waiting time as patients will spend less time on the day they are collecting their repeat scripts.

Community clubs are a good idea because they will help to avoid overcrowding and reduce patient waiting times. Additionally, this will address the shortage of staff and the issue of stigma among HIV/AIDS patients. (Sister-in-charge)

\section{Alleviate staff shortages and workload}

The changeover to implement adherence clubs in communities was also viewed as a strategy that will translate 
to a reduction in the workload for the few clinical staff in a growing health system. This will also translate to a reduction in workload, which could improve the quality of services received at the health facility. These participants indicated that this will also lead to nurses getting more time to focus on currently clinical unstable patients.

Yes, community clubs are a good idea. If stable patients move to the community, nurses can be in a better position to extend their services to those patients that are not stable and initiate new clients on ARVs. (Sisterin-charge)

I think community clubs are a good idea because it would help to reduce the workload of nurses and also benefit patients in terms of travelling. (Club sister)

\section{Antagonist of establishing community- based adherence clubs}

In contrast, some of the participants indicated that the implementation of community-based adherence clubs was not a great idea. Most of the pharmacy personnel expressed concerns in the implementation of the adherence clubs at a community level.

The concerns of individuals who do not favor the implementation of the community-based adherence clubs were categorized as logistical and patient based. The logistical problems were the security of the areas where medication will be distributed, storage conditions of the medication and transportation of the prepacked medication to the distribution sites.

\section{Logistical challenges Security and storage}

In general, pharmacist raised concerns on the implementation of community-based adherence clubs. Some of the participants indicated that security and storage of medication is a priority. One pharmacist responded:

\section{No, I do not think that moving adherence clubs to the com- munity is a good idea due to the concerns I have surrounding the security and storage of medication at a community-based venue. (Pharmacist assistant)}

Some of the pharmacy personnel also raised concern on the transportation of the medication from the health facility to the community venue.

No. I would be worried about how medication would move to the club venue and how uncollected medication will return back to the health facility as security is very important. (Pharmacist)

\section{Stigma and discrimination}

Some of the participants $19(36 \%)$ indicated that the timing to implement community-based adherence clubs will not be ideal as most of the patients that are enrolled in the adherence clubs are largely on ART. Furthermore, participants have mentioned that the community-based adherence clubs will end up being stigmatized or associated with people that are HIV positive. They recommended that the adherence clubs must be implemented in the health facilities until such a time when other chronic patients with diabetes and/or hypertension are picking their medication from the adherence clubs. One of the pharmacists indicated the following:

No. Community-based clubs are not yet a good idea because there is a stigma in ART patients. Patients felt that you (health workers) are discriminating against them when you seclude them. For this reason, such an idea should wait until other chronic patients are included in the clubs. (Pharmacist)

One of the participants indicated that there is a need to empower patients to disclose their status.

No. Community clubs will not be a good idea, not unless patients are empowered to disclose their status. They won't feel comfortable to collect the medication from the community if that is not the case. (Pharmacist)

Another pharmacist also added that the implementation of community-based adherence clubs will fuel stigma as community members will know their status when they pick up the medication from a place outside the health facility.

No, Community clubs will create stigma and patients will not attend because they would not want people to know their status. (Pharmacist assistant)

\section{Increase defaulters}

A data capturer indicated that if the adherence clubs are implemented in community venues, there is likelihood that there would be an increase in the numbers of patients defaulting from picking up their medication. It is further justified that stigma is associated with the pickup point that will be in the communities.

No, I do not think community clubs are a good idea. They will create an increase in the number of defaulters and create stigma because patients will feel exposed. (Data capturer)

Some of the participants also raised concerns that it will be hard to trace the patients that default from their medication as the adherence clubs will be in the communities. The main 
concern by one of the data capturers was around the flow of data from the community to the health facilities. The major worry was the turnaround time from the time the patient misses the appointment date and the information gets to be captured in the tier.net system (a data capturing system that allows for monitoring and evaluation of patient records).

No. Community clubs will not be a good idea because it will be hard for us to keep track of (ART treatment) defaulters.

(Data capturer)

A pharmacist also reiterated that the implementation of the clubs might translate to a high defaulter rate.

No, I do not think community clubs are a good idea they will result in high defaulter rates. (Pharmacist assistant)

Limited patient participation in treatment adherence was one of the factors cited by a respondent. A pharmacist perceived that patients are currently not actively participating in their medication adherence plans, and they are not actively involved in making decisions. The respondent also indicated that the health facility will be in a position to provide a holistic service to the points.

No, I do not think community clubs are a good idea based on the fact that there is a severe lack of patient's participation in adherence. A facility provides a holistic service provision point. (Pharmacist)

\section{Discussion}

This study sought to establish perceived challenges in the movement of adherence clubs from the health facilities to the community-based venues. To our knowledge, it is the first study to explore the perceptions of health facility staff in the transitioning of health facility-based adherence clubs to community-based venues (such as churches, schools, libraries and homes) in South Africa. Key informant interviews have allowed for an in-depth understanding of the perception of the health care workers. Subtleties and complexities about the transition to the community-based clubs were unearthed, which would otherwise have been missed if a positivist enquiry had been adopted.

The study showed that the establishment of the community-based clubs will likely benefit the patients and the health system. The health care workers indicated that the benefits of transitioning clubs from the health facility to the community-based venues include the reduction in the waiting time at the health facilities, reduction in defaulter rate, improvement in adherence to treatment and a reduction in stigma level. The community-based venues will also serve as a convenient site for collection of medication. In some studies, patients have indicated that the benefits of community-based adherence clubs are the reduction of time and costly transportation fees associated with frequent clinic visits. ${ }^{9,19}$

In contrast, some health workers had concerns such as the transportation of the medication from the health facility of the prepacking centers to the community-based distribution sites. One of the major fears stated by most pharmacy personnel was on security related to the theft of ART medication. Studies have shown that ART medication is used as an ingredient in the formation of "Nyaope", a well-known addictive drug. ${ }^{25}$

The concerns of individuals who do not favor the implementation of the community-based adherence clubs were logistical and patient based. The logistical problems were the security of the areas where medication will be distributed, storage conditions of the medication and transportation of the prepacked medication to the distribution sites. This has been inconsistent as some of the participants indicated that the implementation of community-based adherence clubs was not a great idea due to the possibility of an increase in defaulters and loss to follow-up. Contrary to a previous study which found that implementing community-based adherence clubs results in a 35\% reduction in mortality and a $37 \%$ reduction in loss to follow-up when compared with those not attending adherence clubs. ${ }^{5}$

The use of a qualitative approach provided an opportunity to have an in-depth exploration of the issues around implementation of treatment adherence and perceptions of the transition. Unravelling these issues would not have been possible with a quantitative approach. Although this may affect generalizability of the results, qualitative studies tend to provide a detailed information to understand people's behavior of why certain programs are easily acceptable than others, if not the identification of factors influencing nonacceptability. The research involved health worker perceptions, an account of the acceptability of community-based clubs from the health system perspectives, and these might be driven by subjectivity. In addition, the degree of consistency in responses provided by participants is reassuring and/or might affect the reliability of the study results.

The study will inform the processes and planning involved in the decanting of health facility clubs. Consultation and consent need to be sought from patients prior to sending them to the communities. With regard to future research, there needs to be an in-depth analysis between linking to the central dispensing unit to deliver medication and validation of community club registers by health facilities. Furthermore, researchers should also consider further research on the 
identification of secure venues, which are closer to the patient homes. There is a need to establish a working relationship with the health facilities that will lead to adoption and validation of the adherence club registers used in communities. For ease of entry and establishment of the adherence clubs at a community level, there is a need to build a relationship with community structures such as ward counselors, communitybased organizations and ward-based outreach teams.

The major limitation of the research methodology has been that rigor was difficult to maintain, assess and demonstrate. Thus, experienced research conducting the key informant interviews is needed. This research was also not directly involved in the day-to-day program implementation. Future research should look at the perceptions of adherence club patients.

\section{Conclusion}

The transitioning clubs from the health facility to the community venues will be beneficial to the patient and the health system in general. However, there is a need to take precautionary measures so as to ensure that the patients received quality services and stay adherent to medication even when they received their medication from the community venue.

\section{Acknowledgments}

The authors would like to acknowledge Dr Susan Nzenze for her input in editing the manuscript and health facility members who participated in the study in Mpumalanga and Gauteng Province in South Africa.

\section{Author contributions}

NT, OM, JAY, OAA, MSM, KM and PSN have made substantial intellectual contributions in terms of the conception and design of the study. NT, OM and JAY have been additionally responsible for the acquisition and analysis of data. NT, OM, JAY, OAA, MSM, KM and PSN contributed to the interpretation of results and drafting of the article. All authors read and approved the final version of the manuscript.

\section{Disclosure}

The authors report no conflict of interest in this work.

\section{References}

1. Statistics South Africa. South Africa. Mid-year population estimates 2015. 2015. Statistical release P0302.

2. Phokojoe M. National adherence guidelines for HIV, TB and noncommunicable diseases. In: 7th South African AIDS Conference; June 13-15, 2015; Durban, South Africa.

3. Grimsrud A, Sharp J, Kalombo C, Bekker LG, Myer L. Implementation of community-based adherence clubs for stable antiretroviral therapy patients in Cape Town, South Africa. J Int AIDS Soc. 2015;18:19984.
4. Tshuma N, Muloongo K. Missing scheduled appointments among chronic disease patients. In: 7th South African AIDS Conference; June 13-15, 2015; Durban, South Africa.

5. Fatti G, Meintjes G, Shea J, Eley B, Grimwood A. Improved survival and antiretroviral treatment outcomes in adults receiving communitybased adherence support: 5-year results from a multicentre cohort study in South Africa. J Acquir Immune Defic Syndr. 2012;61(4):50-58.

6. Campion EW. Treating millions for HIV - the adherence clubs of Khayelitsha. N Engl J Med. 2015;372(4):301-303.

7. Luque-fernandez MA, Van Cutsem G, Goemaere E, et al. Effectiveness of patient adherence groups as a model of care for stable patients on antiretroviral therapy in Khayelitsha, Cape Town, South Africa. PLoS One. 2013;8(2):e56088.

8. Horwood CM, Youngleson MS, Moses E, Stern AF, Barker PM. Using adapted quality-improvement approaches to strengthen communitybased health systems and improve care in high HIV-burden sub-Saharan African countries. AIDS. 2015;29(Supp1 2):S155-S164.

9. Bemelmans M, Baert S, Goemaere E, et al. Community-supported models of care for people on HIV treatment in sub-Saharan Africa. Trop Med Int Health. 2014;19(8):968-977.

10. Aziz M, Smith KY. Challenges and successes in linking HIV-infected women to care in the United States. Clin Infect Dis. 2011;52(Supp1 2): S231-S237.

11. Mburu G, Ram M, Oxenham D, Haamujompa C, lopenda K, Ferguson L. Responding to adolescents living with HIV in Zambia: a social-ecological approach. Child Youth Serv Rev. 2014;45:9-17.

12. Winestone LE, Bukusi EA, Cohen CR, Kwaro D, Schmidt NC, Turan JM. Acceptability and feasibility of integration of HIV care services into antenatal clinics in rural Kenya: a qualitative provider interview study. Glob Public Health. 2012;7(2):149-163.

13. Shisana O, Rehle T, Simbayi LC, et al. South African National HIV Prevalence, Incidence and Behaviour Survey, 2012. Pretoria, South Africa: HSRC Press; 2014.

14. Mahomed OH, Asmall S, Freeman M. An integrated chronic disease management model: a diagonal approach to health system strengthening in South Africa. J Health Care Poor Underserved. 2014;25(4): 1723-1729.

15. Mills EJ, Nachega JB, Bangsberg DR, et al. Adherence to HAART: a systematic review of developed and developing nation patient-reported barriers and facilitators. PLoS Med. 2006;3(11):e438.

16. Schultz AS, Temple B, Gibbons C, Preston J, Ronson G. Listening to those who are living with HIV and tobacco dependence and exploring their health care context. J Assoc Nurses AIDS Care. 2014;25(1): 46-59.

17. World Health Organization. Antiretroviral Therapy of HIV Infection in Infants and Children: Towards Universal Access: Recommendations for a Public Health Approach-2010 Revision. Geneva: World Health Organization: 2010

18. Amanyire G, Wanyenze R, Alamo S, et al. Client and provider perspectives of the efficiency and quality of care in the context of rapid scale-up of antiretroviral therapy. AIDS Patient Care STDS. 2010; 24(11):719-727.

19. Duff P, Kipp W, Wild TC, Rubaale T, Okech-ojony J. Barriers to accessing highly active antiretroviral therapy by HIV-positive women attending an antenatal clinic in a regional hospital in western Uganda. J Int AIDS Soc. 2010;13:37.

20. Jaffar $S$, Amuron B, Foster $S$, et al. Rates of virological failure in patients treated in a home-based versus a facility-based HIV-care model in Jinja, southeast Uganda: a cluster-randomised equivalence trial. Lancet. 2009;374(9707):2080-2089.

21. Nozaki I, Dube C, Kakimoto K, Yamada N, Simpungwe JB. Social factors affecting ART adherence in rural settings in Zambia. AIDS Care. 2011;23(7):831-888.

22. Lowenthal ED, Jibril HB, Sechele ML, Mathuba K, Tshume O, Anabwani GM. Disclosure of HIV status to HIV-infected children in a large African treatment center: lessons learned in Botswana. Child Youth Serv Rev. 2014;45:143-149. 
23. Tshuma N. An evaluation of the barriers to integrating nutrition, HIV and AIDS in programmes. Manag Perspect A J Manag Stud. 2010;8: 50-69.

24. Mate KS, Ngubane G, Barker PM. A quality improvement model for the rapid scale-up of a program to prevent mother-to-child HIV transmission in South Africa. Int J Qual Health Care. 2013;25(4):373-380.
25. Grelotti DJ, Closson EF, Smit JA, et al. Whoonga: potential recreational use of HIV antiretroviral medication in South Africa. AIDS Behav. 2014;18(3):511-518.

Patient Preference and Adherence

\section{Publish your work in this journal}

Patient Preference and Adherence is an international, peer-reviewed, open access journal that focuses on the growing importance of patient preference and adherence throughout the therapeutic continuum. Patient satisfaction, acceptability, quality of life, compliance, persistence and their role in developing new therapeutic modalities and compounds to optimize clinical outcomes for existing disease states are major areas of interest for the journal. This journal has been accepted for indexing on PubMed Central. The manuscript management system is completely online and includes a very quick and fair peer-review system, which is all easy to use. Visit http://www. dovepress.com/testimonials.php to read real quotes from published authors.

\footnotetext{
Submit your manuscript here: http://www.dovepress.com/patient-preference-and-adherence-journal
} 